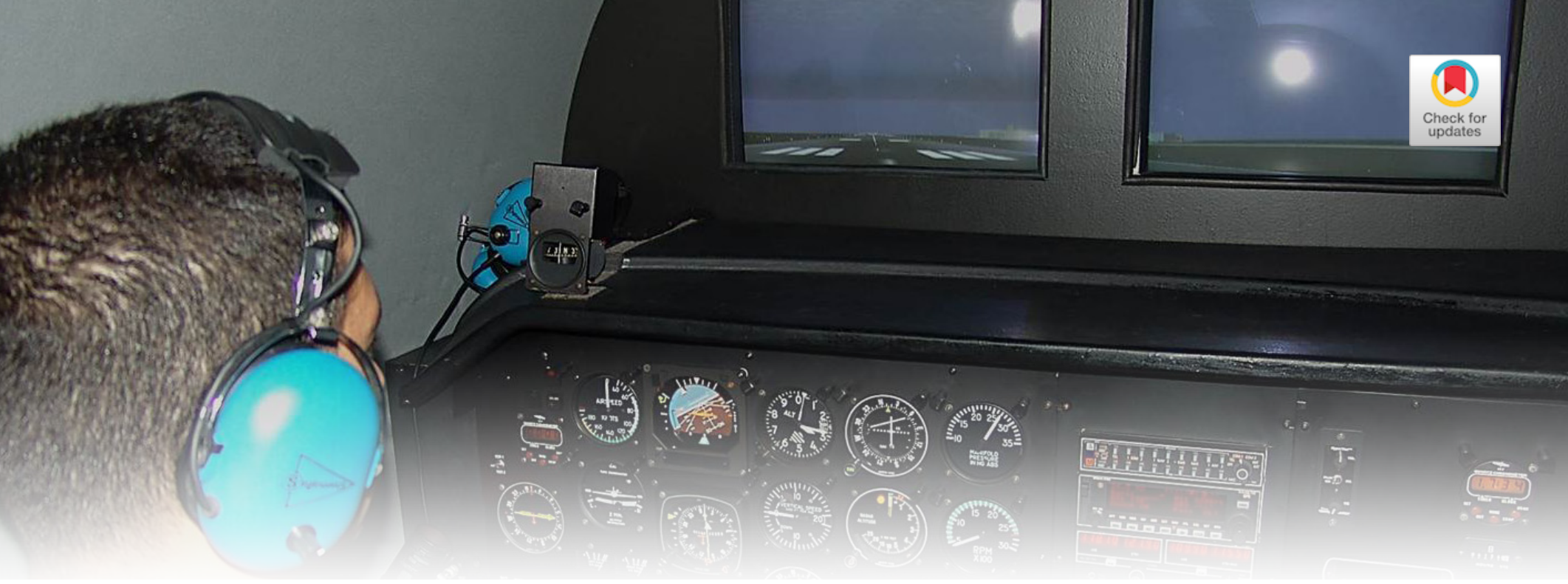

\title{
Simuladores para entrenamientos en la Fuerza Aérea Colombiana
}

\author{
Simulators for Training in the Colombian Air Force ${ }^{2}$
}

\author{
José Fernando Sizza Moreno 3
}

CIENCIA Y PODER AÉREO

ISSN 1909-7050 | E-ISSN 2389-2468 |Volumen 9 | Enero-Diciembre de 2014 | Colombia | Pp. 135-141

Recibido: 20/02/2014

Aprobado evaluador interno: 08/09/2014 Aprobado evaluador externo: 17/10/2014

'Este es un artículo de reflexión derivado del proyecto de investigación: diseño de ambientes de simulación y emulación de escenarios aplicados en juegos de guerra para la Fuerza Aérea Colombiana - FAC, de la línea de investigación - Investigaciones Operacionales de la Maestría en Ciencias Militares de la Escuela de Postgrados de la Fuerza Aérea Colombiana - EPFAC

2 This is a reflection article derived from the research project: designing of simulation environments and emulation of scenes applied in war games for the Colombian Air Force, belonging to the research area- Operational Research of the Master in Aeronautic Military Sciences, of the Graduate School of the Colombian Air Force.

${ }^{3} \mathrm{Mg}(\mathrm{c})$ en Docencia Universitaria - Universidad de Aconcagua (Chile), ingeniero electrónico - Universidad Autónoma de Colombia, Docente en investigación de la Escuela de Postgrados de la Fuerza Aérea Colombiana, tutor virtual de la Universidad Militar Nueva Granada, docente de ingeniería electrónica Universidad San José, docente de metodología de la investigación Corporación Internacional para el Desarrollo Educativo - CIDE. Correo electrónico: jose.sizza@epfac.edu.co MSc in University Teaching - University of Aconcagua (Chile), electronic engineer - Autonoma University of Colombia, Research professor of the Graduate School of the Colombian Air Force, virtual tutor of Nueva Granada Military University, Professor of electronic engineering at San José University, Professor of methodology of research at International Corporation for Educational Development.E-mail: jose.sizza@ epfac.edu.co
Resumen: La simulación es una técnica que compromete ingeniería de software, ingeniería mecánica e ingeniería electrónica, además de emulación, que definen los modelos matemáticos para dotar a una máquina especificaciones funcionales, para la apariencia en tiempo real de comportamientos idénticos a los de su contraparte a imitar. Asimismo, emular es una reproducción de las operaciones de otro, intentando igualarlas e incluso excederlas. De esta manera, una máquina se dota con la simulación y la emulación en tiempo real, diseñando máquinas capaces de imitar y remedar otras, sin los riesgos, humanos o de infraestructura que involucra en la realidad, con la emulación acorde con la máquina a igualar. El entrenamiento puede ejecutarse en este tipo de máquinas, para proporcionar y evaluar las habilidades necesarias a desarrollarse en la vida real, con el fin de disminuir los costos y los tiempos que implican. Es así que el objetivo de este artículo es proporcionar una visión sobre simuladores aeronáuticos puestos en práctica en la Fuerza Aérea Colombiana.

Palabras clave: Emulación, simulación, simulación en la Fuerza Aérea Colombiana.

Abstract: Simulation is a technique that involves software engineering, mechanical engineering and electrical engineering, as well emulation, which defines mathematical models to provide a machine with functional specifications for real-time operation of identical behavior to its counterpart to imitate. Moreover, to emulate is a duplication of the operations of something, trying to match them and even to exceed them. In this way, a machine is provided with simulation and emulation in real time, designing machines able to imitate and mimic others, without the risks, human or of infrastructure involved in reality, with the emulation according to the machine to equal. Training can be done on these machines, to provide and evaluate required skills to be developed in real life, in order to reduce costs and times implied. Thus, the objective of this article is to provide an insight into aviation simulators implemented in the Colombian Air Force.

Key Words: Emulation, Simulation, Simulation in the Colombian Air Force. 


\section{Introducción}

Dentro de la Maestría en Ciencias Militares Aeronáuticas (MACMA) que desarrolla la Escuela de Postgrados de la Fuerza Aérea de Colombia (EPFAC) se cumplen actividades de investigación para solucionar problemas de las diferentes Unidades de la Fuerza Aérea Colombiana (FAC) desde sus dos líneas de investigación, Doctrina Militar Aeroespacial e Investigaciones Operacionales. Esta última, con tres (3) ejes temáticos: estrategia, operaciones y sistemas de simulación, estructurando alternativas temáticas para solucionar dichos problemas.

Al referirnos en el eje temático "sistemas de simulación", es necesario percibir los alcances actuales de la Fuerza en este sentido para determinar qué simuladores hacen falta tanto en la parte operativa como en la parte de gestión, con el fin de establecer propuestas por parte de los estudiantes de la MACMA para obtener simuladores apropiados acorde a un tema en particular o poder ejecutar las soluciones propuestas por los estudiantes en los simuladores actuales en sus opciones de grado.

Además, poder establecer la aparición de estos sistemas de simulación en el mundo obedeciendo a la necesidad del hombre para reducir accidentes, tiempo y dinero por entrenamientos en aeronaves reales, poner de manifiesto las ideas de investigación que se tuvieron en cuenta en esos momentos para poder crearlos, tanto para la parte civil como la parte militar enfocándose en la Fuerza Aérea de los Estados Unidos (USAF, por sus siglas en inglés).

\section{Método}

Para el desarrollo del presente artículo se desarrolló una investigación de enfoque cualitativo, de tipo descriptiva y documental, a fin de conocer la actividad que lleva a cabo la Fuerza Aérea Colombiana en la implementación de simuladores, en el ejercicios de entrenamientos y puesta en marcha, bien sea, en el desarrollo de sus funciones o en respuesta a la asignación de misiones.

\section{Entrenamientos de las Fuerzas Militares}

En todas las esferas laborales, la adquisición de habilidades, destrezas, conocimiento, así como el resultado acertado de tareas, se lleva a cabo, mediante el planteamiento de objetivos claros, como lo estima Bompa (2007) "lo más importante de las tareas de entrenamiento para nóveles y profesionales es la determinación de objetivos asequibles planificados en base a las habilida- des individuales" (p. 17) para una labor determinada, por medio de entrenamientos de larga o corta duración, estos últimos hacen referencia a la enseñanza de prácticas relacionadas con las tareas que se desean ejercitar y así obtener los aprendizajes necesarios para una labor en particular. Luego que, estos aprendizajes ya adquiridos satisfacen las insuficiencias presentes en los puestos de trabajo ya sea por personal nuevo o antiguo y de manera paulatina las insuficiencias futuras se prevén según la preparación que sea necesaria en el personal, como lo indica Silíceo (2004) "la capacitación es la función educativa de una empresa u organización por la cual se satisfacen necesidades presentes y se prevén necesidades futuras respecto de la preparación y habilidad de los colaboradores" (p. 25).

De esta manera, los entrenamientos de las Fuerzas Militares, son preparados con el fin de adquirir habilidades, destrezas, conocimientos y resultados de tareas militares en particular, mejorándolas y consolidándolas para que no sea solo el logro de nuevas habilidades, puesto que, de acuerdo con Lipman (2001) "la educación no debe ser la mera adquisición de habilidades cognoscitivas, sino su mejora y su consolidación" ( $p$. 34). De esta forma, se obtiene personal más eficiente, capaz y asertivo, ya sea en la evaluación de alternativas o en la toma de decisiones en los campos de batalla o de guerra, para lograr ventajas competitivas; hacerlo de una manera eficaz y eficiente $y$, obtener, mantener y mejorar el desempeño global (Chavéz, 2003), y conlleva a tener personal militar de alta calidad para desarrollar tácticas, operaciones o estrategias más adecuadas en el momento indicado en la campaña, batalla o guerra, que permite analizar las mejores técnicas a utilizar en el desarrollo de cualquier operación militar por medio de las experiencias vivenciadas en la academia.

Es necesario destacar que para lograr una alta calidad en el personal preparado, estos entrenamientos deben retribuir a las necesidades naturales del ser humano, convirtiéndose en un recurso de motivación al interior de la empresa (Castillo, 2006). Por lo tanto, el personal militar se siente preparado a la hora de desarrollar una operación militar, con mejor actitud que contagia al resto de personal con los que comparte la misma misión.

Es así como, en la Fuerza Aérea Colombiana (FAC) las prácticas de táctica militar aeronáutica, operaciones militares aeronáuticas y estrategia militar aeronáutica, entre otras, se realizan en la Escuela de Suboficiales de la Fuerza Aérea Colombiana (ESUFA), en la Escuela Militar 
Aeronáutica (EMAVI) y en la Escuela de Postgrados de la Fuerza Aérea Colombiana (EPFAC), donde se dan las prácticas necesarias en cada una de estas actividades. Como resultado de esto; altos estándares de entrenamiento, con los costos financieros, logísticos y humanos que puedan generar. Del mismo modo y en su gran mayoría las prácticas de guerra, se desarrollan en la Escuela Superior de Guerra (ESDEGUE), y al interior de los estados mayores conjuntos y de fuerzas.

Las prácticas en actividades de seguridad y defensa nacional desarrolladas en la ESDEGUE son previstas en el Simulador Táctico Operacional Conjunto (SITOC) el cual cuenta con doctrina y simulación de varios escenarios adaptados a la realidad de las Fuerzas Militares de Colombia (FF.MM). Este simulador permite realizar ejercicios ajustados a las necesidades del programa académico a que se aplique en la toma de decisiones operacionales, estratégicas o análisis de crisis, por medio del trabajo conjugado de los comandantes, facilitándoles la práctica y el entrenamiento siendo partícipes como comandantes, miembros de estados mayores y gerentes en el planteamiento, conducción y ejecución de planes operacionales y estratégicos previamente elaborados.

Ahora bien, la enseñanza de estas actividades se evidencia en los diferentes programas impartidos en cada una de estas escuelas, y se determina la efectividad del proceso educativo con la didáctica de enseñanza aprendizaje que se utiliza, para influenciar sobre los estudiantes y en las metas que persigue para un aprendizaje en particular con la utilización de herramientas tecnológicas (D'agostino, 2007), valiéndose de estándares de apoyo en el uso de la tecnología para la enseñanza, el aprendizaje y la evaluación de los estudiantes (C.I.D.E., 2003), determinando la forma más eficaz de tener personal militar aeronáutico con preparación de calidad en cada una de las labores a desarrollar en la FAC y contribuyendo competentemente con la misión y la visión de la misma en un entorno de supremacía del espacio aéreo colombiano dando cumplimiento al artículo 216 de la constitución política colombiana.

Como lo señala Fernández (2001) "la efectividad requiere considerar tanto la eficacia como la eficiencia, es la combinación de ambos. Sabemos que se puede ser muy eficaz pero sin eficiencia, y también es frecuente en educación ver acciones eficientes pero ineficaces" ( $p$. 317) luego que, determinar hasta qué punto se es eficaz pero al mismo tiempo determinar la eficiencia, queda en papel del proceso de autoevaluación, de esta ma- nera se entrega al personal nuevos conocimientos que no tengan falencias en la eficacia o en la eficiencia, ya que al determinar esas faltas tanto de eficacia como de eficiencia a tiempo, se corrigen de manera adecuada, lo que implica que el estudiante debe hacer la evaluación de su propio trabajo y comprometerse con un plan personal de recuperación que corrija las faltas señaladas (Castillo, 2002). De esta forma, se aprende holísticamente, logrando que el aprender sea alojado de manera permanente en la memoria del estudiante (Sperling, 2004), así los estudiantes entregan su mayor capacidad al momento de entrar en el desarrollo de una operación aeronáutica a cualquier nivel.

En cuanto a la utilización de herramientas tecnológicas, sea una de estas la simulación de sistemas, considerada como una valiosa técnica en la enseñanza (Martín, 2008), desafiando de esta manera a dichas escuelas en abrir el abanico de opciones para la enseñanza de los saberes en el campo militar aeronáutico, por medio de sistemas reales simulados con infraestructura tecnológica de punta, entregando el máximo de experiencia permitido a los alumnos según sus disciplinas en ambientes reales, mediante la simulación de sistemas antes de ser puestos en una aeronave o el desarrollo de un proceso (FAC, 2010) dentro del sistema de calidad de la FAC.

Las opciones de enseñanza dentro de una academia siempre deben estar acompañadas de los sentidos, en una interacción, destacándose oído y vista (Blackwood, 2011), proporcionando a los estudiantes un proceso más eficiente y eficaz al involucrar la mayor cantidad de sentidos en su aprendizaje. De esta manera (Blackwood, 2011), se puede esperar el máximo desempeño de los militares de la FAC, ante las diferentes condiciones en las cuales se encuentren involucrados.

\section{Simuladores de vuelo}

Al abordar el tema de simuladores, cabe precisar qué es un simulador. Según Guasch (2009) es la "técnica que permite imitar (o simular) en un ordenador el comportamiento de un sistema real o hipotético según ciertas condiciones particulares de operación" (p. 1). De igual modo, los simuladores consisten en una interacción persona - computador con "programas que representan un modelo o entorno dinámico y en el que a través de gráficos o animaciones facilitan al estudiante la visión de lo que ocurre en el entorno que está simulando" (Pérez, 2001, p. 276). Así pues, cada estudiante se 
siente inmerso en el equipo a operar como si estuviera en la realidad, ya que al verse rodeado de la interactividad virtual del mundo que lo involucra en el escenario, le va "aportando flexibilidad y sobre todo, interactividad a la enseñanza" (Murcia, 2004, p. 67). De esta manera, se hace más efectiva la enseñanza y el aprender del estudiante, al el momento de una operación aeronáutica en todos los niveles, dependiendo de la experiencia adquirida y la función en la que se desenvuelva él estudiante (simulador de operaciones).

Actualmente, existen diferentes tipos de simuladores según su fin. Se cuentan con los a) simuladores de conducción, para enfrentarse con mayor seguridad en la clases prácticas (Monclus, 2010); b) simulador de carreras (autos, motocicleta, camión, entre otros); c) simulador de vuelo (Senge, 2006), para dominar el mundo aeronáutico. Por otra parte, existen: d) simulador de trenes; e) simulador clínico médico, utilizado por los estudiantes en medicina y paraclínico; f) simulador de negocio, para evidenciar entornos empresariales; g) simulador musical; $h$ ) simulador termo - solar, i) simulador de seguridad cibernética "para el adiestramiento de personas y grupos encargados de salvaguardar la seguridad cibernética de gobiernos e infraestructuras militares y civiles críticas" (Infodefensa, 2012, párr.1). Es evidente que por cada actividad que se enseña en la vida real se puede utilizar un simulador, destacándose las actividades de mayor riesgo con el fin de evitar posibles accidentes humanos, riesgos en infraestructura, con los elevados costos que estos acarrean.

En este sentido, se comprende, que con los simuladores de vuelo inicialmente se pretendía evitar accidentes por falta de conocimiento de las aeronaves a utilizar, sin ser aerotransportados sus usuarios. A manera de ejemplo, tenemos; el "Sander Teacher" en 1910 (Riley, 2008). Éste consistía en un aeroplano que tenía la posibilidad de sentir el aire inclinándose y girando libremente. Para la misma década se crea un simulador de vuelo simplemente con un barril montado en una mesa; posteriormente, en 1930 el "Link Trainer" (Riley, 2008) simulaba movimientos mecánicos con instrumentos de control, preparando pilotos para la Segunda Guerra Mundial; el "Celestial Navigation Trainer" de 1943 (Kirk, 2009) tenía la posibilidad de albergar la tripulación de un bombardero capacitándola en misiones nocturnas.

Ahora bien, con el apogeo de la creación de los primeros computadores, se da la invención de los primeros simuladores electrónicos y en 1948 (Rolfe, 1979) para la aerolínea Pan American, se crea en Curtiss-Wright el simulador B377 Stratocruiser con todos los sistemas utilizados por una aeronave comercial, adaptando un sistema de cámaras para proyectar imágenes de los alrededores del aeropuerto a un televisor. Un sistema parecido fue utilizado por los militares para simular bombardeos. Para los años 60 en la General Precision INC (livibe, 2013) se desarrolla un simulador con 10 movimientos, lo que llevó a que en 1977 se crearan, con todas las prestaciones necesarias, para un piloto de aerolínea.

Desde ese momento, los mayores avances se dieron en tecnologías de visualización como lo indica Hidalgo (2011) en su libro Israel del siglo XXI Tradición y Vanguardia, dando testimonio de la solvencia israelí, en el caso de las vistas del exterior de la cabina, que se realizaban utilizando un espejo esférico que las proyectaba, con lo cual mejoró la sensación de vuelo. Finalizando así, adelantos tecnológicos esenciales en la construcción de simuladores de vuelo, que establecieron los estándares de diseño para ser retomados por especialistas en software, electrónica, y mecánica entre otras disciplinas en ingeniería para que sean las desarrolladoras de nuevos conceptos en la materia.

Ante esta perspectiva, un nuevo concepto en estos días consiste en un compartimiento que efectúa movimientos reales desarrollando los conocimientos de nuevas disciplinas y procedimientos aeronáuticos, cuenta con una cabina real abierta con mandos, panel y pantallas para desarrollo de un vuelo simulado; conjuntamente cuenta con un compartimiento de instructor, donde se pueden considerar las variables de vuelo, aeronave, meteorología en tiempo real, entre otros, a ser experimentados por el alumno que percibe varias impresiones de una operación aérea, además de conectarse con el exterior apoyados en modernos cascos que permiten la conexión con internet.

En este orden de ideas, se desarrollan actividades de entrenamiento militar en condiciones de simulación, y se reducen los costos financieros, logísticos y humanos por entrenamiento y capacitación al personal militar, como es el caso de los simuladores ubicados en las diferentes unidades militares de la Fuerza Aérea Colombiana (Ver Tabla 1). Con éstos se pretende aumentar la capacidad de reacción de cada uno de los cadetes u oficiales según sea el caso, además poder evaluar las diferentes capacidades de atención y manipulación de los controles emulados (Simulador OV10 ubicado en EMAVI), propiciando enfrentamientos 
entre equipos (azul y rojo) confirmando los diferentes trabajos que se realizan entre equipos de aeronaves inscritas a la FAC explicándolos, transformándolos y analizándolos para poder ser ejecutados en la realidad de forma más eficaz, confirmando los procesos que se ejecutan en los cielos de Colombia, lo cual contribuye a cumplir con la misión de la FAC.

Tabla 1. Simuladores de vuelo en las diferentes unidades operacionales de la Fuerza Aérea Colombiana.

$\begin{array}{ccc}\text { CANTIDAD } & \text { TIPO } & \text { UNIDAD } \\ & & \\ 2 & \text { HAWK } & \text { EMAVI } \\ 1 & \text { T-27 } & \text { CACOM-2 } \\ 1 & \text { F-28F } & \text { CACOM-4 } \\ 2 & \text { UH-1H } & \text { CACOM-4 } \\ 2 & \text { HUEY II } & \text { CACOM-4 } \\ 1 & \text { Frasca 242-T } & \text { CATAM } \\ 1 & \text { UH-60 } & \text { CACOM-4 }\end{array}$

Fuente: elaboración del autor.

Se hace necesario, destacar los simuladores de vuelo de helicópteros en el Comando Aéreo de Combate No.4. CACOM 4 ubicado en Melgar (Tolima), los cuales han capacitado aproximadamente a 4171 hombres y mujeres entre FAC, Armada, Ejército, Policía y Fuerzas extranjeras, siendo considerados como pioneros en capacitaciones de Latinoamérica (Colprensa, 2013, p. 28-II). No obstante, es importante que se establezca innovación, aplicación y apropiación de tecnología al interior de los programas de entrenamiento de las Fuerzas Militares, de esta manera se incide positivamente en dicho proceso (Cañal de León, 2002), De ahí que se cuenta con un sinnúmero de posibilidades de capacitación en el marco de la innovación y renovación pedagógica (Cañal de León, 2002). Es decir, que la calidad de la educación o la libertad de la enseñanza, se entiende como un "conjunto de ideas, procesos y estrategias, más o menos sistematizados, mediante las cuales se trata de introducir y provocar cambios en las prácticas educativas vigentes" (p. 11).-

Conforme sucede esto, se puede estar igual o mejor entrenados que países avanzados, permitiendo la apreciación del conflicto o post-conflicto de manera permanente y reconocida, y demostrando un alto nivel de preparación, por lo que se justifica plenamente el reconocimiento del hecho (Duvauchelle, 1994). En este sentido, se deben tener en cuenta las caracterís- ticas de los modelos de simulación y los modelos de emulación, para ser implementados y de esta manera seguir fortaleciendo las Fuerzas Militares, así el "fortalecimiento de frente a los grupos al margen de la ley, mediante la adecuada dotación y profesionalización de las fuerzas armadas" (Fernández de Soto, 2004, p. 470) indudablemente, proporciona unas Fuerzas Armadas preparadas, entrenadas para enfrentar los desafíos que le atañen por parte del actuar del país, de igual manera, preparados para enfrentar escenarios sociales disociadores que se viven actualmente en Colombia, donde son necesarias las Fuerzas Militares para conservar su legitimidad y no como afirmara el vicepresidente de Colombia Francisco de Paula Santander al presidente libertador Simón Bolívar: el pueblo se encuentra trastornado por las Fuerzas Militares (1928).

\section{Ambientes de simulación en el marco de la defen- sa nacional}

Respecto a las características técnicas que se deben tener en cuenta para realizar una adecuada capacitación, una de estas es la utilización de escenarios debidamente adaptados en los cuales se logre una emulación propicia, donde herramientas como el mapa real de Colombia con todos sus accidentes geográficos, es fundamental para tener una mejor habilidad. Asimismo, agregar los mandos incluidos en las aeronaves reales, hacen que se pueda adaptar mejor según la necesidad operativa, buscando también que el tripulante se sienta confortable al ubicarse en el simulador, al encontrarse éste dotado con la realidad necesaria para ser operado. Paralelamente, debe ser capaz de ejecutar todas las órdenes necesarias con el mismo comportamiento que se obtiene en el mundo real, con la misma información y a un costo factible.

De igual manera, los Ambientes de Simulación en Escenarios Militares (ASEM) en las FF.MM de todos los países del mundo, establecen un marco de entrenamiento militar crucial en el actual mundo globalizado, capacitando a las unidades militares, mediante los mejores recursos que se encuentren en el mundo, así los simuladores ejercen un cambio en el entrenamiento militar. Como es el caso de la USAF, que crea la Air Force Agency for Modeling and Simulation (AFAMS, por sus siglas en inglés), partiendo de la base de la soberanía nacional, y entregando la directiva No 5000.59 donde es explícito que se debe capacitar en adquisición, prueba, análisis, planeación, experimentación, entrenamiento educativo y formal (Directiva 5000.59, 2007) a las unidades militares basados en modelado y simulación. 
En Colombia, preservar la soberanía del territorio nacional es fundamental para dar cumpliendo al artículo 217 de la Constitución Política Nacional, el cual establece que "Las Fuerzas Militares tendrán como finalidad primordial la defensa de la soberanía, la independencia, la integridad del territorio nacional y del orden constitucional.", para cumplir este artículo, se desarrollan actividades encaminadas a la defensa nacional y enmarcada en las misiones de todas las fuerza; una de estas actividades se establece en el entrenamiento regular de las fuerzas, involucra recursos económicos y utiliza las mejores alternativas encontradas a nivel nacional y mundial, tomando los entrenamientos que utilizan un simulador para desarrollar la velocidad de reacción y la actuación técnica táctica en condiciones de déficit (Platanov, 2001), como uno de los soportes en entrenamiento más económicos ya que se sustentan en la utilización del mismo recurso por los diferentes integrantes de las Fuerzas sin las necesidades, costo e infraestructura que esto conllevaría, debido a la utilización de armamento, gasolina o personal calificado que no podría ser utilizado en los lugares donde son necesarios.

De esta manera, el rubro por entrenamiento militar desarrollado en ASEM, toma escenarios militares de posibles misiones que se basan en lecciones aprendidas, en los cuales interactúan oficiales de la Fuerza Pública con estrategias militares, tácticas militares, de gestión de operaciones militares o de gestión militar que los lleven a ganar una campaña, batalla o guerra emulada, y propicia conclusiones que pueden ser puestas en el escritorio para ser ejecutadas con una mejor planeación de cada una de las unidades a ser utilizadas que no se tuvieron en cuenta en las primeras planificaciones antes de ser ejecutadas por los oficiales de las fuerzas en el simulador, provee una mejor capacitación que instruyéndolos por medio de lecturas basadas en campañas exitosas y no exitosas que se han dado a nivel nacional y mundial en la historia, para que sean adaptadas en el campo de batalla según las necesidades de cada uno de los comandantes en algún momento, desafiándolos a que sean recordadas para ponerlas en práctica. De este modo, es mejor actuar con todos los sentidos, integrándolos por medio de un simulador.

Es así como, dentro de las características técnicas de los modelos de simulación se encuentran las siguientes:

- Deben ser completos.

- Adaptables.

- Que tengan credibilidad.
- Deben ser simples (menor número de parámetros).

- Con factibilidad tanto en información como en recursos.

- Económicos.

\section{Conclusiones}

LOS ASEM que actualmente están instalados en Colombia, no establecen una competencia significativa en el entrenamiento de oficiales de la FAC, debido a que no se emulan escenarios en los cuales se puedan establecer tácticas aeronáuticas, estrategias aeronáuticas y operaciones aeronáuticas en entrenamiento militar, aéreo, de procesos gerenciales, de procesos de apoyo y de gestión pública, donde se logre efectuar planeación, conducción y coordinación de cada una de las operaciones descritas, debilitando el entrenamiento que se instruye a cada uno de ellos y los cuales no pueden entregar lo mejor de sus capacidades. Debido a la falta de coordinación de cada una de las unidades militares puestas a trabajar en equipo, bien sea, por no poder operar una máquina en particular o ante un grupo que tiene la necesidad de ser dirigidos en los campos de batalla, pero no son asertivos ante las órdenes entregadas para su ejecución en un lugar específico, sin tener que dejar de lado las funciones que están ejecutando por el hecho de encontrarse mal dirigidos.

Ahora bien, un ASEM con estas características mencionadas anteriormente, y puestas al servicio de los integrantes de la Fuerza Aérea Colombiana con las especificaciones funcionales necesarias para poder entrenar el personal involucrado en las operaciones militares, aéreas, de procesos gerenciales, procesos de apoyo y de gestión pública, en los cuales puedan efectuar la planeación, conducción y coordinación de estas, contribuirá a ejercer con mayor dominio una operación militar aeronáutica efectiva, contundente en escenarios reales sin los costos financieros, logísticos y humanos acarreados por adiestramientos reales, que puedan eliminar el error por las repeticiones que se hagan de la misma misión, cumpliendo de esta forma con el art 217 de la Constitución y la misión de la Fuerza, corroborado por la visión de la FAC que establece tener el mejor personal y capacitarlo para este fin. Así, se puede contratar los servicios de instalación y puesta en marcha de más simuladores que contengan emulador de todas las aeronaves de la Fuerza, capacitando ya sea en pilotaje, atención de las mismas hasta gestión aeronáutica. 
Por consiguiente, las especificaciones funcionales deben ser modelos matemáticos que se basen en cada una de las necesidades por parte de las disciplinas de los oficiales de la Fuerza Aérea Colombiana, ayudado de una infraestructura que se comporte según la necesidad de la respectiva disciplina del oficial/alumno, como por ejemplo un prototipo de avión Kfir, en el cual se involucren los movimientos reales al operar la aeronave, o establecer las pautas necesarias para efectuar las gestiones que deben tener en cuenta por estar al servicio de una misión y las necesidades a suplir en ésta, ya sea táctica, operacional o estratégica, con cada una de las capacidades de la Fuerza establecidas en un simulador; finalmente poder ser destinadas a un fin general, operación exitosa por cada uno de los alumnos/ oficiales, con la experiencia generada por la utilización del simulador en cuanto a planificación en la obtención del objetivo que se debe lograr por parte de los integrantes de la Fuerza.

\section{Referencias}

Blackwood, R. (2011). El poder de la predicación y de la enseñanza. [En línea] Disponible en http://books.google.com.co/books?id= PLg8UN1BcH4C\&printsec=frontcover\&dq=El+poder+de+la+p redicaci\%C3\%B3n+y+de+la+ense\%C3\%B1anza\&hl=es\&sa=X\& $\mathrm{e}=$ =_Sh_VKCNLYmPyAS_s4HYBg\&ved=0CBoQ6AEwAA\#v=one page\&q=El\%20poder\%20de\%20la\%20predicaci\%C3\%B3n\%20 y\%20de\%20la\%20ense\%C3\%B1anza\&f=false.

Bolivar, S. (1828). Colección de documentos relativos a la vida pública del libertador de Colombia y del Peru Simon Bolivar. Caracas: Devisme.

Castillo, J. (2006). Administración de personal: un enfoque hacia la calidad. Bogotá: Ecoe ediciones LTDA.

Castillo, S. (2002). Compromisos de la evaluación educativa. Madrid: Pearson Education.

Cañal de Leon, P. (2002). La innovación educativa. España: Akal S.A.

Centro de Investigación y Documentación Educativa-CIDE. (2003). La integración de las nuevas tecnologías en los centros. Una aproximación multivariada. Madrid: FER.

Coss Bu, R. (2008). Simulación un enfoque práctico. México: Limusa. Chávez, G. (2003). Manual para el diseño de sistemas de calidad basado en competencias laborales. México: Panorama.

D’agostino de Cersósimo, G. (2007). Aspectos teóricos de la evaluación educacional. San José de Costa Rica: EUNED.

Department of Defense United States of America-DoD. (8 August, 2007). DoD Directive 5.000.59, Modeling and Simulation (M\&S) Management. Arlington, Virginia: Department of Defense United States of America. In: http://www.dtic.mil/whs/ directives/corres/pdf/500059p.pdf

Duvauchelle, M. (1994). Las fuerzas armadas y carabineros de Chile: su regulación constitucional y orgánica constitucional. Chile: Jurídica de Chile.
Escuela de Guerra. (2013). Centro de Simulación y Análisis de Crisis (CESAC). Bogotá: Escuela de Guerra.

Fernández de Soto, G. (2004). La ilusión posible: un testimonio sobre la política exterior colombiana. Bogotá: Norma.

Fernández, S. (2001). Análisis de efectividad en la evolución de programas sociales y educativos. Revista De educación. Disponible en http://dialnet.unirioja.es/servlet/ articulo?codigo $=19380$

Fuerzas Armadas de Colombia, FAC. (2005). Manual de Operaciones Aéreas (O-MAOPA).Bogotá: Imprenta de las Fuerzas Militares.

Fuerzas Armadas de Colombia, FAC. (2010). Plan estratégico institucional 2011-2030. Bogotá: Imprenta de las Fuerzas Militares.

Hidalgo, A. (2011). Israel, Siglo XXI Tradición y Vanguardia. La Coruña: Gesbiblo, S.L.

Infodefensa. (2012). Elbit Systems desarrolla un nuevo simulador de seguridad cibernética.

España: Autor. [En línea] Disponible en http://www.infodefensa. com/es/2012/06/05/noticia-elbit-systems-desarrolla-unnuevo-simulador-de-seguridad-cibernetica.html.

Kirk, D. (2009). Words to Measure a War: Nine American Poets of World War II. North Carolina: McFarland \& Company, Inc., Publishers.

Lipman, M. (2001). Pensamiento complejo y educación. Madrid: De la Torre.

Martín, E. (2008). Aprendizaje por simulación en la enseñanza de aviación. EE.UU: Createspace.

Monclus, J. (2010). La seguridad víal en las empresas. Programas internacionales de promoción. Madrid: Mafre.

Murcia, J. H. (2004). Redes del saber. Investigación virtual, Proceso educativo y autoformación integral. Bogotá: Cooperativa editorial Magisterio.

Pérez, M. (2001). Sistemas de interacción persona-computador. España: Servicio de impresiones de la Universidad Castilla de la mancha.

Platonov, V. N. (2001). La preparación física. Barcelona: Paidotribo.

Ríos, D. (2000). Simulación Métodos y aplicaciones. Bogotá: Alfaomega.

Riley, R. H. (2008). Manual of simulation in healthcare. New York: Oxford.

Ross, S. M. (1997). Simulación. México: Prentice Hall

Senge, P. M. (2006). La quinta disciplina en la práctica. Buenos Aires: Ediciones Granica S.A.

Siliceo, A. (2004). Capacitación y desarrollo de personal. México: Limusa.

Sperling, A. P. (2004). Psychology made simple. New York: Cía. General de ediciones S.A. de C.V.

Stark, J. (2010). Motion - is there a requirement in large fixed-wing aviation simulators? Canadian forces college JCSP 36. [En línea] Disponible en http://www.cfc.forces. gc.ca/259/290/296/286/Stark.pdf

Para citar este artículo: Sizza, J. (2014). Simuladores para entrenamientos en la Fuerza Aérea Colombiana. Ciencia y Poder Aéreo, Vol. 9 (1). Pp. 135-141 